\title{
Videocolaboração nas ações de Televisita no Hospital Militar de Área de São Paulo (HMASP) para apoio familiar no combate ao COVID-19: estudo de caso
}

\author{
Mauricio M. M. Segobia \\ Telessaúde São Paulo DIS \\ Universidade Federal de São Paulo \\ (UNIFESP)São Paulo, SP \\ segobia@unifesp.br
}

\author{
Guido Lemos de Souza Filho \\ LAVID/Universidade Federal da \\ Paraíba (UFPB) João Pessoa, PB \\ guido@lavid.ufpb.br
}

\author{
Claudia Galindo Novoa \\ Telessaúde São Paulo DIS \\ Universidade Federal de São Paulo \\ (UNIFESP) \\ claudia.novoa@unifesp.br \\ Jane de Almeida \\ Labcine/Universidade Mackenzie \\ São Paulo, SP \\ segobia01@unifesp.br
}

\author{
Cicero Inácio Silva \\ Telessaúde São Paulo DIS \\ Universidade Federal de São Paulo \\ (UNIFESP) \\ cicero.silva@unifesp.br \\ Marcelo de Melo Marcon \\ Exército Brasileiro/Hospital Militar \\ de Área de São Paulo, São Paulo, \\ secinfo@hmasp.eb.mil.br
}

\begin{abstract}
This article analyzes the experience of using a video collaboration tool, through the virtual visit (Televisita) strategy, at the São Paulo Army's Hospital (HMASP). The objective is to analyze the impact of the use of a system developed especially for this project, to reflect on the psychosocial effect that the virtual visit (Televisita) plays on patients and family members affected by COVID-19 and who are hospitalized in the HMASP facilities. The article also assesses and explores some strategies for using virtual visits that may be used in the Brazilian Army's HMASP and also describes future potentials in relation to video collaboration in digital health.
\end{abstract}

\section{KEYWORDS}

Telessaúde, Televisita, Telemedicina, Videocolaboração Saúde Digital.

\section{Telessaúde no HMASP}

Segundo a Organização Mundial de Saúde (OMS), a disponibilização de serviços de saúde, onde a distância é um fator crítico, utilizando TICs (tecnologias de informação e comunicação) para a troca de dados válidos para diagnóstico, tratamento e prevenção de doenças, terapias; onde contribui- se para o avanço da saúde define o termo telemedicina (1). No Exército Brasileiro já testou-se várias possibilidades de uso da telemedicina $(2,3,4,5)$. Mais precisamente no HMASP, já vem-se adotando essa prática, realizando teleconsultas em campanha e até mesmo transmissão de cirurgias ao vivo (6). Recentemente, no maior exercício de adestramento das tropas do Comando Militar do Sudeste -

In: VII Workshop "O Futuro da Videocolaboração" (WCT-Video 2020), São Luís, Brasil. Anais Estendidos do Simpósio Brasileiro de Sistemas Multimídia e Web (WebMedia). Porto Alegre: Sociedade Brasileira de Computação, 2020. (C2020 SBC - Sociedade Brasileira de Computação.

ISSN: 2596-1683
Operação Agulhas Negras - foi desdobrado um Hospital de Campanha na cidade de São João da Boa Vista, e este foi conectado por telessaúde (teleconsultas e telemonitoramento), estando a mais de $700 \mathrm{~km}$ de distância com o HMASP, no intuito de levar remotamente atendimento médico especializado a distância para as tropas empregadas no exercício $(7,8)$. A questão central neste estudo refere-se à possibilidade do uso da telessaúde para práticas de televisitas para pacientes internados no HMASP acometidos com COVID-19.

\section{Videocolaboração e Televisita}

Devido à pandemia do novo coronavírus (COVID-19), os pacientes internados no Hospital Militar de Área de São Paulo (HMASP), diagnosticados com o novo coronavírus não podem receber visitas. Com isso, a apreensão dos próprios pacientes e familiares mais próximos é muito grande, o que torna a situação ainda mais delicada. Visando a humanização hospitalar e amenização desse quadro, as Subcomissões de Psicologia e Informática (equipes técnicas criadas pelo HMASP no intuito do enfrentamento à COVID-19), elaboraram um projeto para a implantação da Televisita para estes pacientes. Com a regulamentação da telemedicina no Brasil (9), foi possível colocar em prática esta iniciativa. Para tal, foram definidos os protocolos de atendimento, utilização de tablets e celulares, e, junto à Rede Nacional de Ensino e Pesquisa, em parceria com o LAVID da Universidade Federal da Paraíba e com a Universidade Federal de São Paulo (UNIFESP), o aplicativo de telemedicina chamado Vídeo For Health (V4H) permitiu o encontro virtual entre os pacientes, familiares e equipe assistencial de saúde do HMASP. 


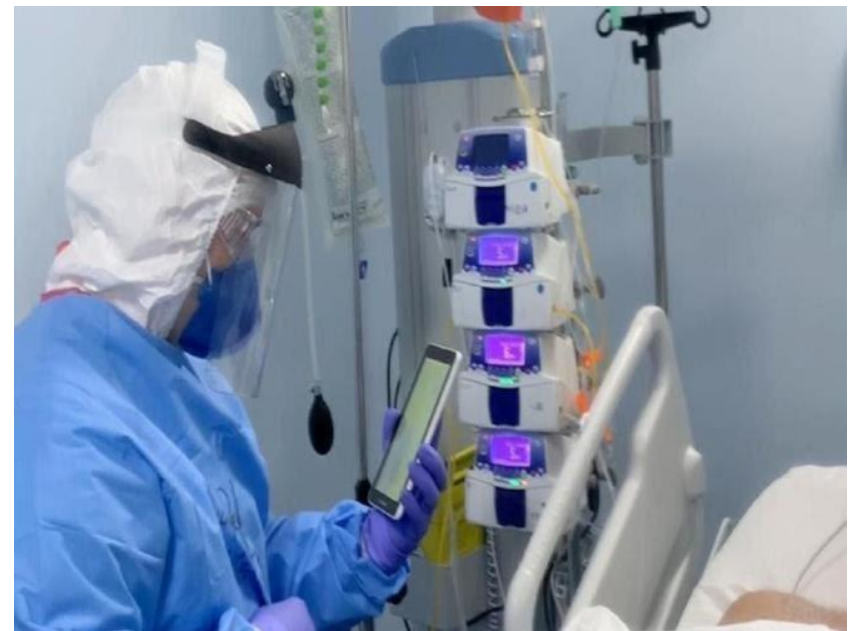

Figura 1: Televisita à beira-leito no HMASP.

\section{Avaliação Psicosocial da Videocolaboração no HMASP}

Neste sentido, em março de 2020, O Hospital Militar de Área de São Paulo (HMASP) recebeu o ambiente de Televisita desenvolvido pelo GT-V4H da Rede Nacional de Ensino e Pesquisa (RNP), no qual foram desenvolvidas várias ferramentas customizadas para o agendamento de visitas virtuais dos familiares aos pacientes acometidos pela COVID- 19 internados no HMASP. O sistema desenvolvido pelo GT-V4H é uma ferramenta de teleatendimento por meio de vídeo, no qual dois ou mais participantes podem interagir em um ambiente seguro por meio do vídeo. A ferramenta dispõe de ambiente de chat, gravação e permite que o ambiente seja compartilhado com mais pessoas, permitindo que vários familiares possam participar ao mesmo tempo de uma mesma visita, mesmo estando em localidades remotas em diferentes pontos. A primeira Televisita ocorreu no mês de abril de 2020, próximo da declaração de situação de calamidade pública no Brasil. O procedimento da televisita foi acompanhada por profissionais da área de saúde mental e médicos intensivistas do HMASP, nos mesmos moldes agendamento de uma visita presencial, mas dessa vez, utilizando-se das TICs para o contato visual, no intuito de aproximar os pacientes internados (acometidos por COVID) e seus familiares. Um dos resultados dessa prática dáse em ,além de conversar com o médico sobre o estado do paciente, poder vêlo e tirar dúvidas, e além disso, as famílias ainda têm todo o apoio da equipe de psicologia $(10,15)$.

\section{Dados referentes a televisita no HMASP}

Foram efetuadas 105 consultas via Televisita, que proporcionaram aos pacientes e aos familiares momentos de acolhimento no período de pandemia, foram atendidas 34 famílias e cada Televisita era composta por 3 visitantes com o tempo médio em cada visita de aproximadamente 20 minutos, reservando 10 minutos para o boletim médico e 10 minutos para a visita e explicação médica, caso houvesse a necessidade. As visitas foram realizadas em uma escala de 3 a 4 vezes por semana e toda essa ação em conjunto da Tecnologia da Informação e o Serviço de Saúde trouxe aos familiares momentos emocionantes, pois podeser observar a diminuição da ansiedade das famílias ao poderem ter contato com seus entes queridos.

\section{Infraestrutura do $\mathrm{V} 4 \mathrm{H}$}

Para o estabelecimento da plataforma HMASP Vídeo foram necessárias 4 (quatro) máquinas virtuais com sistema operacional Ubuntu com as seguintes especificações: 8 GB RAM, 30Gb Disco Virtual, placa de rede com conexão Gigabit, Acesso via SSH. Essa infraestrutura mínima garante 2 (duas) gravações simultâneas de conferências. Todas as máquinas virtuais do core do serviço possuem IPs públicos e acessíveis na Iinternet, com conexão estável e de alta velocidade com a Web para melhor qualidade na teleconsulta. Para fazer gravações, 1TB de armazenamento provido via NFS , snedo montado em todas as máquinas virtuais utilizadas para o serviço de gravação. Vale salientar que cada hora de conferência gravada ocupa um espaço de aproximadamente 700 MB em disco. Após a instanciação da infraestrutura necessária, realizou-se a instalação e execução dos serviços que compõem a plataforma HMASP Vídeo e suas respectivas dependências. Após realiza-se o download do app V4H (Play Store ou App Store), e através de autenticação de usuário e senha, entra no ambiente do HMASP Vídeo utilizando um tablet ou smarphone.

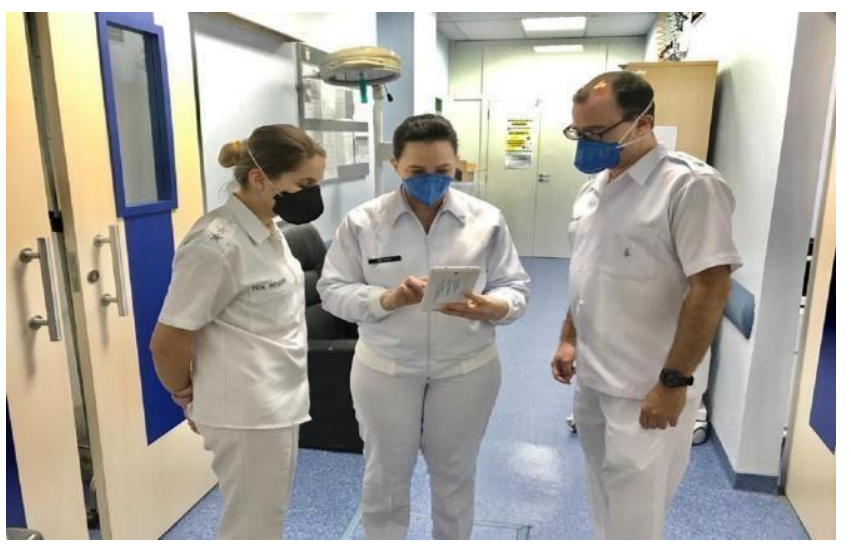

Figura 2: Profissionais da equipe de psicologia comunicando-se previamente com familiares.

\section{Visão de Futuro}

Conectar diversas organizações militares com o HMASP no intúito de apoiá-las com atendimento especializado de forma remota utilizando a telemedicina. Difundir o conceito de telessaúde no âmbito da Força Armada. Levar médicos especialistas nas regiões mais distantes e fronteiriças de nosso País. 
Videocolaboração nas ações de Televisita no Hospital Militar de Área de São Paulo (HMASP) para apoio familiar no combate ao COVID-19: estudo de caso

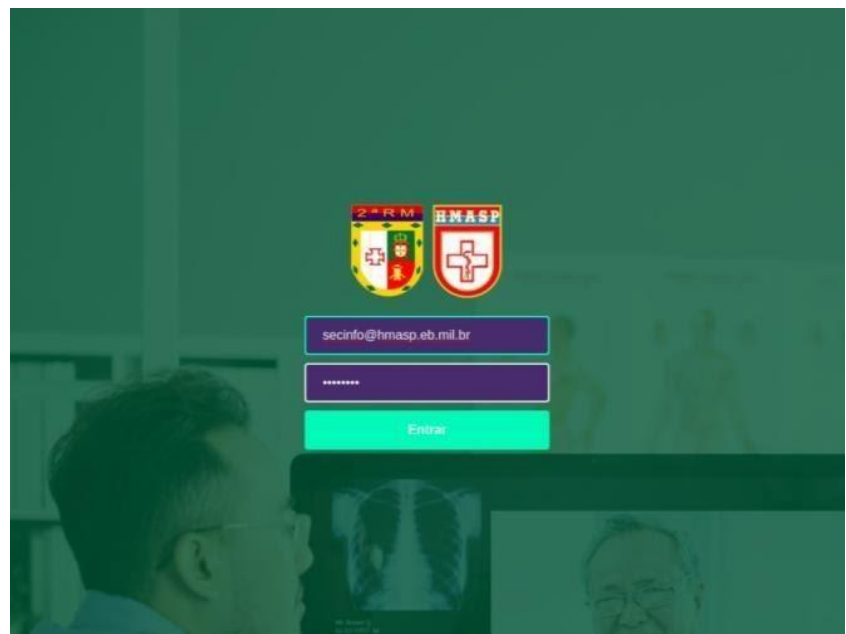

Figura 4: Ambiente V4H Cloud criado para as televisitas no HMASP (hmasp.v4h.cloud)

\section{Conclusão}

O uso dos recursos disponibilizados pelas redes avançadas e pelas tecnologias de transmissão de imagem em ultra-alta definição têm cada vez mais possibilitado inúmeras pesquisas e ações práticas no campo da telemedicina, telessaúde e educação permanente em saúde. Desta forma, a entrega da saúde pode ser transformada e aprimorada, possibilitando feitos inéditos beneficiando tanto os pacientes, quanto os profissionais de saúde envolvidos no processos assistencial.

\section{AGRADECIMENTOS}

Agradecemos à Direção, equipes de Saúde Digital e Tecnologia da informação do HMASP, do Telessaúde São Paulo Unifesp, do Grupo de Pesquisa em Saúde Digital: Telessaúde e Tecnologias da Informação da Unifesp (http://dgp.cnpq.br/dgp/espelhogrupo/1179489123867343) e do GT-V4H da RNP, coordenado pelo Prof. Dr. Guido Lemos.

\section{REFERENCIAS}

[1] KHOURI, S. Telessaúde: análise da sua evolução no Brasil. 2003. Dissertação (Mestrado em Fisiopatologia Experimental) - Faculdade de Medicina, Universidade de São Paulo, São Paulo, 2003. Acesso em: 20-11- 2018.

[2] Telemedicina é mais um projeto inovador que chega no Hospital das Forças Armadas, 2020, Exército Brasileiro, 2020 - Disponível em: $<$ https://tinyurl.com/y6hq2z9q $>$. Acesso em: 1 de jun. de 2020 .

[3] Teste de telemedicina no Hospital de Guarnição de São Gabriel da Cachoeira traz resultados positivos, Exército Brasileiro, 2020. Disponível em $<$ https://tinyurl.com/y536235g $>$ Acessado em 10 Ago 2020

[4] Projeto Telemedicina - Parceria com a Universidade Federal Fluminense na busca de atendimento médico à distância. Exército Brasileiro, 2017. Disponível em: $<$ https://tinyurl.com/y45cgkrk $>$. Acesso em: 20 de jun. de 2020.

[5] Exército Brasileiro apoia testes de telemedicina em Iauaretê e São Gabriel da Cachoeira-AM, Exército Brasileiro, 2020. Disponível em $<$ https://tinyurl.com/yyjs9v6a> Acessado em 30 JUL 2020..

[6] Neurocirurgia do Hospital Militar de Área de São Paulo é a primeira a utilizar implante inédito no Brasil, Exército Brasileiro, 2019. Disponível em < https://tinyurl.com/yxp3sgn9>. Acessado em: 1 Jun 2020.
Anais Estendidos do WebMedia'2020, São Luís, Brasil

[7] HMASP, pela primeira vez na OPAN, Hospital Militar de Área de São Paulo 2020. Disponível em: <https://tinyurl.com/yxww5u8t> Acessado em Jun 2020.

[8] Profissionais de saúde simulam transporte de feridos durante o Exercício Agulhas Negras, 2019, Exército Brasileiro. Youtube TV CMSE. Disponível em: $<$ https://tinyurl.com/yx6y9twg > . Acesso em: 08 de out 2020. 0:01:30.

[9] BRASIL. Lei no 13.989, de 15 de Abr. de 2020. Dispõe sobre o uso da telemedicina durante a crise causada pelo coronavírus (SARS-CoV-2)., Brasília,DF, Abril 2020.

[10] Hospital inaugura televisita, Exército Brasileiro, 2020. Disponível em: $<$ https://tinyurl.com/yxweajo5> Acessado em Jun 2020.

[11] SOARES DE OLIVEIRA, S et al. Experiência de Uso da Arthron na Transmissão de Cirurgias em Tempo-Real para Telessaúde, Programa de Pós-Graduação em Informática, Universidade Federal da Paraíba, João Pessoa, 2012.

[12] BRAGA JUNIOR, W. Estratégia para armazenamento e recuperação de experiência multimídia em telessaúde: um estudo de caso no Projeto Ambiente de Vídeo Colaboração em Saúde (GTAVCS). 2013. 151 f. Tese (Doutorado em Informática) - Universidade Federal da Paraí•ba, João Pessoa, 2013.

[13] BRITO, T.D . Análise da colaboração nos Grupos de Interesse Especial (SIG) da Rede Universitária de Telessaúde (RUTE). 2016. 108f. Dissertação (Mestrado em Ciências) - Escola Paulista de Medicina, Universidade Federal de São Paulo. São Paulo, 2016.

[14] DA SILVA, C.I. et al. Análise dos processos videocolaborativos do Núcleo Telessaúde Brasil Redes Unifesp na Atenção Básica. In: ROESLER, V. \& CIUFFO, L. (Orgs.) O Futuro da Videocolaboração: Perspectivas. Porto Alegre: SBC, 2017

[15] SHAW, S.E. et al. Video Consultations Between Patients and Clinicians in Diabetes, Cancer, and Heart Failure Services: Linguistic Ethnographic Study of Video-Mediated Interaction. J Med Internet Res 2020;22(5):e18378. DOI: $10.2196 / 18378$ 